\title{
Open Science at the Science-policy Interface: Bringing in the Evidence?
}

\author{
Stefan Reichmann and Bernhard Wieser
}

Author Contact Details: stefan.reichmann@tugraz.at; bernhard.wieser@tugraz.at

\begin{abstract}
Part of the current enthusiasm about Open Science stems from its promises to reform scientific practice in service of the common good, to ensure that scientific outputs will be found and reused more easily, and to enhance scientific impact on policy and society. With this article, we question this optimism by analysing the potential for Open Science practices to enhance research uptake at the science-policy interface. Science advice is critical to help policy makers make informed decisions. Likewise, some interpretations of Open Science hold that making research processes and outputs more transparent and accessible will also enhance uptake of results by policy and society at large. However, we argue that this hope is based on an unjustifiably simplistic understanding of the science-policy interface that leaves key terms ("impact", "uptake") undefined. We show that this understanding - based upon linear models of research uptake - likewise grounds the influential "evidence-policy gap" diagnosis which holds that to improve research uptake, communication and interaction between researchers and policymakers need to be improved. The overall normative stance of both discussions has side-lined empirical description of the science-policy interface, ignoring questions about the underlying differences between the policy domain and academia. Importantly, both Open Science and literature on closing the evidence-policy gap recommend improving communication (either in terms of the content or the means) as a viable strategy. To correct some of these views, we combine insights from policy theory with a systematic review of the literature on the evidence-policy gap in the health domain and find that removing barriers to access by itself will not be enough to foster research uptake.
\end{abstract}




\section{Introduction: The role of science in policy}

Whereas in earlier decades, public servants played a dominant role in policy advice, they are now expected to consult external sources (academia, stakeholder organizations, think tanks, political organizations) (Head, 2015). At least in industrialized countries, governments increasingly rely on external advice and (scientific) evidence as a way to improve governance (Hermann et al., 2015). This has led to increased interest in understanding the interlinkages between policy making and expertise (Lundin and Öberg, 2014). The goal of research in government is to find information that will help to solve specific, predefined policy problems in real time (Glied, Wittenberg and Israeli, 2018). While the Evidence-Based Policy (EBP)-movement was instrumental in promoting rigorous analysis of policy options and programs (Head, 2016), a salient issue in the literature concerns the fact that topical scientific expertise is not being used in respective policy decisions despite its availability (Haines, Kuruvilla and Borchert, 2004; Graham et al., 2006), a problem that has been labelled the "evidencepolicy gap" (Haines, Kuruvilla and Borchert, 2004) and has been singled out as a major obstacle for reaching the UN's sustainable development goals in public health (Panisset et al., 2012).

In this paper, we explore the ways in which Open Science could bridge the evidence-policy gap. In fact, utilization of publicly available scientific results and data by policymakers has been claimed to be one of the benefits of Open Science (Tennant, Jacques and Collister, 2016; Olesk, Kaal and Toom, 2019). Likewise, a variety of measures have been proposed for researchers who wish to make an impact on policy makers. Like advocates of Open Science, analysts of the evidence-policy-gap recommend improving communication and other forms of interaction between researchers and policymakers to close the gap. We argue that inquiring into the role of Open Science in policy making can be regarded as a variant of the more general issue of research utilization or research uptake in policy making. Open Science thereby acts as a lens through which the science-policy interface can be studied by asking: How would scientific policy advice be improved if we improved scholarly communication? To this end, we reviewed literature studying the ways in which knowledge enters the policy process as well as literature documenting the reforms to scholarly communication and research practice envisioned by Open Science.

To the best of our knowledge, science advice has been most comprehensively problematized in the health policy domain, under the heading of an evidence-policy gap (Brownson and Jones 2009; Cairney, Oliver, and Wellstead 2016; Choi et al. 2016; Dodson, Geary, and Brownson 2015; Ellen et al. 2018; Gollust, Seymour, Pany, Goss, et al. 2017; Haynes et al. 2011; Oliver et al. 2017). This literature identifies concrete barriers to research utilization, offering suggestions to researchers how those barriers could be overcome. Yet, the relationship between policymakers and researchers at the science-policy interface is rarely scrutinized and frequently described as one of mutual misunderstanding or outright mistrust (Haynes et al., 2012), where researchers and policy makers are incapable of successful communication.

In this article, we briefly review the history of the science-policy interface to interrogate the potential for Open Science practices to enhance scientific policy advice while paying attention to the fact that the science-policy interface already involves inputs from outside academia. In addition, bringing knowledge into the policy process requires trust and (established) relationships, which has led to the development of dedicated knowledge brokers (Pielke, 2007) tasked with translating and synthesising results. We describe the development of the science-policy interface through theoretical and empirical work to question whether making scientific communications more transparent and open is enough to foster effective scientific policy advice. We identify an important overlap between the recommendations to reform science in the spirit of openness respectively policy advice - both favour reforming scholarly communication - and go on to investigate what Open Science contributes in terms of enhancing research uptake, conceived as a participatory process. Following Cairney's (2016) observation that descriptions of the evidence-policy gap largely remain a-theoretical, we analyse implicit policy models present in the reviewed literature to argue that the linear conception of research uptake hampers moving beyond the "barriers and enablers"-rhetoric of much of this work. We spell out the implications for the prospects of Open Science practices to make an impact on scientific policy advice, arguing for a more nuanced understanding of Open Science as participatory research. 
The paper begins with a brief exposition of the development and tenets of the Open Science movement. It then describes the history of the use of scientific evidence in policymaking and the development of our understanding of the science-policy interface. Based on a systematic literature review, we go on to discuss early linear models of the science-policy interface in some detail to argue that a large proportion of recent empirical work on research uptake has failed to take into account the changing landscape of science advisory systems by focussing (almost) exclusively on the relationships between researchers and policy makers. We then make a case that while Open Science practices may be helpful if we assume a linear model of the science-policy interface, the complexity of current science advisory systems entails that Open Science needs to move beyond a narrow conception of reforming scientific communication if is to positively affect scientific policy advice. Proponents have taken for granted the premise that academia is in need of top-down reform, a claim that has been couched in the superficially positive terminology of "openness", without clearly stating in what way science was ever "closed".

This work is based upon and significantly extends a project deliverable prepared for the European Commission under Grant Agreement No. 824612 (ON-MERRIT - Observing and Negating Matthew Effects in Responsible Research and Innovation Transition) (Reichmann et al. 2020). For the deliverable, a systematic literature review was performed in SCOPUS and Web of Science. Articles were selected based on abstract and keywords, with additional hand-selection of articles from the sample so-generated based on references to (aspects of) Open Science. Results were then summarized, validated, and synthesised. The relevant literature was restricted to (1) all (peer-reviewed) materials pertaining to policy making, (2) all materials referring to (aspects of) OS within that corpus. For the analysis, a triaging strategy was used (similar to the approach described in Contandriopoulos et al., 2010) to keep the amount of text manageable. The obtained data are narrative (i.e. in the form of published research articles), so the primary analysis strategy was narrative as well. Combining a summative approach and an analytical approach, we produced a synthesis document of how the literature describes research uptake. We included a total of 115 articles in the review. 


\title{
A brief exposition of Open Science
}

\author{
Which problem is Open Science meant to solve?
}

As both policy circles and researchers are increasingly embracing Open Science, questions of how to make scientific practices more open and transparent are the subject of ongoing debate (Albornoz et al., 2017). The term Open Science encompasses a variety of meanings ranging from publicizing research outputs - Open Access in its various forms - to making all aspects of the research process accessible (Fell, 2019), including data (e.g. Giffels, 2010), notebooks, analysis plans, and code (Ibanez, Avila and Aylward, 2006; Ram, 2013) as well as research evaluation and peer review (Shanahan and Olsen, 2014; Ross-Hellauer, 2017). Open Science denotes a bundle of practices and associated ideas such as accessibility, reproducibility, (data) sharing, and collaboration (Vicente-Saez and Martinez-Fuentes, 2018), but is often used interchangeably with Open Access and Open Data (Albornoz et al., 2020). Open Access is considered a gatekeeper for institutions to adopt other Open Science practices, e.g., by the Association of European Research Libraries (LIBER) (Albornoz et al., 2017). In many respects, arguments in favour of Open Access might resonate most with the aims and needs of research institutions, so adopting Open Science practices very frequently starts with adopting an Open Access policy. Thus the Budapest Open Access Initiative (2001):

"An old tradition and a new technology have converged to make possible an unprecedented public good. The old tradition is the willingness of scientists and scholars to publish the fruits of their research in scholarly journals without payment, for the sake of inquiry and knowledge. The new technology is the internet. The public good they make possible is the world-wide electronic distribution of the peer-reviewed journal literature and completely free and unrestricted access to it by all scientists, scholars, teachers, students, and other curious minds. Removing access barriers to this literature will accelerate research, enrich education, share the learning of the rich with the poor and the poor with the rich, make this literature as useful as it can be, and lay the foundation for uniting humanity in a common intellectual conversation and quest for knowledge. For various reasons, this kind of free and unrestricted online availability, which we will call open access, has so far been limited to small portions of the journal literature." (http://www.budapestopenaccessinitiative.org/read)

The above excerpt identifies access barriers as the primary motivation for "opening" science. If only, the argument goes, scholarly literature was freely accessible, humanity would be united in its "quest for knowledge". Of course, this is predicated on the assumption that removing barriers by itself is not enough; rather, scholars need to be willing to share their data. This second assumption has spawned a vast literature studying the motivations (not) to share data (Piwowar, 2011; Piwowar and Vision, 2013a). The statement further highlights acceleration, assuming that the speed with which research is published is an issue. We will return to both assumptions below. In short, then, Open Science practices have been associated with better and more efficient science (Leonelli, Spichtinger and Prainsack, 2015), but also with economic growth (Tennant, Jacques and Collister, 2016), and increased transparency of knowledge production (Gilmore et al., 2017). Textbook definitions have characterized Open Science as an umbrella term for a set of practices spanning the entire research cycle, all with the aim of bringing greater transparency and/or participation to research (Fecher and Friesike, 2014; Nosek et al., 2015). Popularizing books such as Michael Nielsen's "Reinventing Discovery" (Nielsen, 2013) have argued that networked science is "democratising" research, listing "increased equity" as a "key success factor" for Open Science. In the same vein, Grahe et al. (2020) claimed that "Open science principles of openness and transparency provide opportunities to advance diversity, justice, and sustainability by promoting diverse, just, and sustainable outcomes".

Yet, as scholars working in postcolonial traditions have pointed out (Hillyer et al., 2017), whatever it is that Open Science criticizes about "closed" science has nothing to do with the myriad postcolonial or feminist critiques of the ways in which even epistemological regimes of science are deeply implicated in society's mechanisms of oppression (Harding, 2002). However, this is not what Open Science (as it stands) seems to be challenging. Rather, Open Science proposes a rather conventional, positivist vision of science, albeit remodelled along the possibilities associated with increasing datafication (Kitchin, 2014; Leonelli, 2016). Openness is therefore much closer to increasing efficiency than it is to inclusivity and access. This is evident in what Open Science considers to be the fundamental problems with contemporary science, most notably, the multiple crises of replication that have swept various fields 
since the 2010s (Leonelli 2018), as well as the publication crisis (Suber, 2012). Indeed, core tenets of the Open Access movement have been thoroughly appropriated by academic publishers by now. If we follow Mirowski (2018), this suggests that the objective of the movement might not be to reform scientific practice, but to remodel it along the lines of "platform capitalism", a perspective that "corresponds to Mosco's critique that big data 'promotes a very specific way of knowing'; it encourages a 'digital positivism or the specific belief that the data, suitably circumscribed by quantity, correlation, and algorithm, will, in fact, speak to us' (Mosco 2015, 206)." (Richterich, 2018, p. 10)

\section{Reforming scientific practice?}

Open Science promotes the accessibility of scientific outputs, but its criticism of "traditional" research practices often remains implicit and has rarely been investigated systematically. Open Science advocates list a standard set of benefits of Open Science practices for researchers, such as increased citation rates (Piwowar, Day and Fridsma, 2007; Piwowar and Vision, 2013b), increased media coverage (McKiernan et al., 2016), more transparent research evaluation (Pöschl 2012; Beck et al. 2018), increased reproducibility (Toelch and Ostwald, 2018), increased control over research outputs through retaining copyright and publishing under Creative Commons (CC) licenses (McKiernan et al., 2016), and establishment of priority via preprints (Vale and Hyman, 2016). Yet, as McKiernan et al. (2016) have pointed out, arguments promoting Open Science policies rarely address the practical barriers involved in changing researchers' behaviours, such as existing incentive structures for research careers (Nosek et al., 2015) or a lack of infrastructures (Amorim et al., 2017; Cox et al., 2017; Bugaje and Chowdhury, 2018). Indeed, Open Science depends heavily on digital infrastructures allowing the free exchange of research outputs (Bardi, Casarosa and Manghi, 2018), which explains Mirowski's (2018) critical review of the role of platforms in Open Science. Open Science principles favour the use of open source software and the publication of all research outputs under open licenses (Fecher and Friesike, 2014). Open Science requires policies regulating data protection, copyright, and licensing (Pisani et al., 2010; Caso and Ducato, 2014; Carroll, 2015; Carrozza and Brieva, 2015) as well as a compatible economic space to develop (Tennant, Jacques and Collister, 2016; critically: Mirowski, 2018). Open Access publishing and the need for suitable business models is a case in point (Suber, 2012). Making scientific outputs freely available requires fundamental changes in the economic system that values and protects intellectual property rights and the privileged access and use of scientific resources and outputs (Ibanez, Avila and Aylward, 2006; Carroll, 2015; Schaeffer, 2019). With respect to the science-policy interface, some (Tennant, Jacques and Collister, 2016 see also the Budapest Open Access Initiative of 2002; Olesk, Kaal and Toom, 2019) have suggested that Open Access facilitates knowledge-utilisation in policy making, a claim that has been criticized on the grounds that accessibility is neither necessary nor sufficient (Owen and Pansera, 2019; von Schomberg, 2019). What matters, then, is how a more open, participatory form of research affects scientific knowledge production in the first place.

\section{Five Solutions and the Scope of Open Science}

Given the heterogeneity of interpretations of Open Science, Fechner and Friesike (2014) suggested systematising the various approaches in terms of five "schools": "The infrastructure school (which is concerned with the technological architecture), the public school (which is concerned with the accessibility of knowledge creation), the measurement school (which is concerned with alternative impact measurement), the democratic school (which is concerned with access to knowledge) and the pragmatic school (which is concerned with collaborative research)" (ibid. p. 17). Following this systematic we argue that influential interpretations of Open Science concern science-to-science interaction and collaboration, while only parts of what sails under the banner of OS addresses scienceto-public relationships and engagement. In essence, the "public school" asks questions as to how science can be open to collaboration by the public (for the most part understood as citizen science) and how 
science can benefit the public. ${ }^{1}$ The proposed benefits of Open Science for various groups (as well as the associated critiques of the "old" system) can be summarized as follows:

1) Quality: Increasing the transparency and reproducibility of scientific production

2) Access: Removal of access barriers

3) The public funding argument

4) Quantity: Acceleration of scientific productivity

5) Relation: Democratization and public engagement

With respect to research uptake, Open Science has indeed been hailed as a panacea to apparently rampant levels of distrust in science among the general populace, evidenced in scepticism vis-à-vis climate change, anti-vaccination movements, the correlation between tobacco smoke and cancer, and GMOs, to name but a few. In an argument vaguely reminiscent of the Public Understanding of Science-movement of the 1980s (Wynne, 1995), Open Science advocates have suggested that the removal of access barriers will serve to boost public trust in science as well as evidence-based policy making, with particular emphasis on enrolling the public:

"The opportunities for transparency, authenticity and timeliness of the record created by open science could both reveal the scientific process in real time and allow claims to be viewed within the context of their underlying data. Open science thus has the potential to contribute to the substantiation of the relationships which are central both to people's trust in science and to science's trust in people. (Grand et al., 2012)"

Others (Stilgoe, 2013) argue, in the same vein, that the trend towards increased openness holds the promise for more public engagement. Some have claimed that Open Science continues a long-standing agenda of fostering participatory research. Civil society actors, users, patients, NGOs, industry and other societal stakeholders are not only said to benefit from open access to scientific outputs (Tennant, Jacques and Collister, 2016), but, crucially, are regarded as resourceful contributors to processes of knowledge production. Therefore, Citizen Science is highly valued in Open Science as furthering public engagement throughout, starting with agenda setting, definition of research questions as well as contributing to data collection and analysis, publication and evaluation of research findings (VicenteSaez and Martinez-Fuentes, 2018) as well as facilitating dialogue between science and society (Leonelli, Spichtinger and Prainsack, 2015).

What has been less frequently examined (at least to our knowledge) concerns an apparent tension between some of the claims. For instance, acceleration of scientific productivity, something many advocates desire seems to counteract the potential for greater transparency and reproducibility, for the simple reason that yet more articles will attract fewer readers and potentially exacerbate the replication crisis. At any rate, the idea that scientific productivity needs a boost is difficult to justify (to say the least) precisely not because of a lack of metrics but because of competing metrics.

In our opinion, the common denominator of all the elements of Open Science discussed above is a reform of scholarly communication in terms of what is communicated resp. shared (everything from lab notebooks to research data), with whom (anyone with a computer with internet access), when (as early in the research process as possible), and how (relying on digital platforms of a certain kind). The promise lies in reforming all these aspects under the auspices of increasing transparency to fend off the upheavals caused by neoliberal reforms of the university (Slaughter and Rhoades, 2010; Münch, 2014). However,

" $[\mathrm{t}]$ he irony of the situation is that although this petrification of the scientific enterprise could largely be attributed to previous neoliberal 'reforms' in the first instance, the remedy proposed is to redouble neoliberal policies, now under the rubric of 'open science'.” (Mirowski, 2018, p. 189)

\footnotetext{
${ }^{1}$ The term "democratic school" is ambiguous in this context, since Fecher and Friesike refer to access to scientific knowledge without having a specific user in mind, rather addressing a generic subject of knowledge (Fecher and Friesike, 2014. p. 25). Knowledge provided is not appropriated by non-scientific audiences and therefore boils down to a science-to-science communication of research outputs.
} 
Concerning the removal of access barriers, this is supposed to apply not only to publications (in the form of scientific articles, say). Rather, Open Science wishes to extend "transparency" to all kinds of research outputs, including research data. However,

"Much of the vision behind [FAIR data] presupposes that scientific data is inherently fungible, once a few pesky obstacles are cleared away. Some outstanding work by Leonelli (2016; Leonelli et al., 2015) has demonstrated that this impression concerning the nature of Open Data is illusory. Partisans of open science love to celebrate the kumbaya of 'data sharing'; Leonelli counters that there is no such thing. Data in the modern context would never venture outside the lab were it not for dedicated curators and their attached data consortia, such as the Open Biology Ontology Consortium (active since 2001). [...] The partisans of open science neglect to highlight the extent to which they define what the data actually signifies in Science 2.0, something that should give pause to anyone believing that data is effortlessly separable from its generators and curators." (Mirowski 2018: 190 f.)

Aside from the loftier normative aspects of Open Science and how they hold up to scrutiny, the technical aspects of it (at least with respect to data and publications) have been thoroughly examined. Excellent contributions by Leonelli and collaborators (Leonelli, 2016) draw attention to a problem for Open Science by suggesting that there are fundamental barriers to sharing (data, in this instance) that cannot be simply removed. The idea that data are inherently shareable if only we manage to remove certain obstacles is flawed; Leonelli's (2016) meticulous ethnographies show that data would rarely be shared beyond their context of origin were it not for the work of data curators.

The upshot of this discussion can be summarized as follows: Open Science is an umbrella term for a program of reforming science through reforming scholarly communication, with a clearly normative thrust (i.e., to make science better). If we follow Fecher and Friesike's (2014) analysis, there are narrower and wider interpretations of Open Science, depending on who is included. The above discussion has shown how many of the hopes of Open Science advocates don't hold up to empirical scrutiny. This concerns the content of its aims as well as the means that are being proposed. In the next section, we wish to prepare our main point - that both Open Science as well as policy advice - frequently fall short of delivering adequate empirical descriptions, in particular as regards the science-policy interface - by describing how theorizing the science-policy interface has developed in the second half of the $20^{\text {th }}$ century. 


\section{A bit of history: Phases of the Science-Policy Interface}

The way in which science and research have engaged with government has undergone a considerable evolution in the second half of the 20th century (Reichmann, Wieser and Ross-Hellauer, 2020). As the science-policy interface evolved, so did conceptual models developed by social and political scientists (Gluckman, Bardsley and Kaiser, 2021). Before World War II, governments were entrusted with defining the common good. Post-war Europe saw increasing numbers of scholars engaging with policy in an era of increased investment in science (Sokolovska, Fecher and Wagner, 2019). Maasen and Weingart (2005) identify several historical developments conducive to the changing relationship between science and policy making: a general push towards democratization in industrialized countries and an associated development of political movements operating outside the formalized political process. From the 1980s onwards, the science-policy interface was increasingly framed with a view towards "society" as deficient (Wynne, 1995), with experts separated from lay people and the incomprehensible language used by scientists as the main obstacle to public understanding of science, with "translation" as the preferred intervention. The corresponding style of communication has been described as the "deficit model" (Wynne, 1993, 1995) characterised by 1) a (knowledge) hierarchy between scientists and their lay audiences, 2) one-way communication from experts to lay people, 3) a focus on textbook knowledge and 4) the expectation that lay people will appreciate science and its achievements (Felt, 2003).

For the US, Weingart $(1983,1999)$ diagnosed increasing formalization of communication between science and policy that he traced to President Eisenhower's 1957 appointment of a science and technology advisor and committee. This model still exists, at least in part (Gluckman, Bardsley and Kaiser, 2021), but has since been supplemented by more sophisticated models of science advice (e.g., Mode-2-Science, cf. Gibbons et al., 1994). Many of these models assume that policy making is a linear process working rationally from problem-identification to -solution and that appeals to scientific data and established facts will lead to better problem characterizations and, by extension, better policy. This linear approach to the science-policy interface has recently come under criticism. It is now understood that the linear model does not adequately reflect the complexity of the science-policy interface (Cairney, 2016) and the processes by which knowledge enters the policy sphere (Jasanoff, 2005). More recent conceptualizations accordingly recognize that knowledge claims are increasingly put forth by various actors with varying degrees of connection to either formalized policy advice, decision makers, or research. In addition, it has been recognized that what types and sources of knowledge are used in policy making depends on the policy context as well (Cairney, 2016).

With respect to the science-policy interface, the role of communicating the results of evidence synthesis falls to the knowledge broker (Gluckman, Bardsley and Kaiser, 2021), as described in Roger Pielke's book The honest broker (Pielke, 2007) where the role is defined as an intermediary at the science-policy interface. Pielke describes four (idealized) types of conduct: 1) the pure scientist, 2) the science arbiter, 3 ) the issue advocate, and 4) the honest broker. The critical point in Pielke's model seems to be the relationship of the issue advocate to research, as the role borders on a lobbying position (Nagasaka, Böcher and Krott, 2016, p. 149).

In a recent review, (Sokolovska, Fecher and Wagner, 2019) describe three broad historical phases the science-policy interface has undergone in the second half of the 20th century, each with its associated policy model: 1) linear models of science-policy advice that roughly correspond to the notion of policymaking as an elite system, 2) interactive models of science-policy interface that roughly correspond to policy-making as a deliberative process (Weiss, 1979), and 3) embedded models of the science-policy interface that are associated with increased attention to inclusion and societal engagement.

Note that the three models are ideal types; they are designed to help guide our theorizing. In that sense, they are not mutually exclusive, i.e., they can (and frequently do) coexist. Each model corresponds to a class of strategies and techniques associated with managing the science-policy interface that researchers (can) adopt. Each phase thereby follows its own logic (Sokolovska, Fecher and Wagner, 2019, who cluster various models of the science-policy interface under each phase). Each model (roughly) corresponds to a distinct historical phase in the development of the science-policy interface following 
World War II. The first phase ("linear models") corresponds to a linear understanding of the sciencepolicy interface, where advice is conceived as a one-way communication process (Sokolovska, Fecher and Wagner, 2019) based on a dichotomy of facts (science) and values (policy). The second phase ("interactive models") is characterized by the assumption that both scientists and policy makers collaborate in a non-hierarchical way in search of the best problem solutions and that policy-making and knowledge production cannot be neatly separated; policy-relevant knowledge is developed in continuous exchange between researchers, policy makers, and societal actors, both with respect to problem definitions as well as defining goals and means. The hitherto final phase ("participatory models") revolves around the question of engaging society at large in the policy process. Participatory models regard the science-policy interface as a discursive process between researchers and policymakers (Sokolovska, Fecher and Wagner, 2019) and are similar to interactive models in this respect; however, they are characterized by intense reflection on "formats of societal engagement and the language of communication on the science-policy interface" (Sokolovska, Fecher and Wagner, 2019), e.g. in terms of changing relationships between academia and the larger society in the triple-helix-model (Etzkowitz and Leydesdorff, 1990), which similarly constitutes a shift away from linear models of the sciencesociety relationship. It should be noted that the linear model of the science-policy (and society) relationship is not an invention of post-war science popularisation (Reichmann, Wieser and RossHellauer, 2020). However, this type of science communication is still around, thereby perpetuating the deficit model (Felt, 2003, p. 19) developed from the 1950s onwards. The following exposition of the three phases of the science-policy interface follows Sokolovska et al. 2019 but considerably modifies the third phase.

The family of linear models is of particular interest to our research aim. In the following sections we analyse a body of empirical work on the science-policy interface that suggests that the science-policy interface can be described as a gap. Based on an in-depth analysis of this literature, we argue that this diagnosis builds upon an ultimately inadequate linear understanding of the science-policy interface. 


\section{Mind the Gap: How research does (not) inform policy}

\section{The role of evidence in policy}

Research into the role of evidence in policy making constitutes a field of considerable breadth. Interestingly, a large proportion of empirical work into the science-policy interface has been relatively unperturbed by the historical dimensions of the phenomenon, sketched in the preceding section. Barriers and facilitators to knowledge transfer are a well-recognised research topic (Mitton et al., 2007, p. 735) with a set of established results. The bulk of the work on knowledge transfer pertains to barriers and facilitators to evidence-based policy making (Lavis et al., 2003; Mitton et al., 2007; Oliver et al., 2014). Academics have strong motivations to give policy advice, both in terms of demonstrating "impact" to funders as well as making a difference to society (Oliver and Cairney, 2019). Boswell $(2008,2009)$ identifies three functions of expert knowledge in policy making (cited after Holm and Ploug, 2015, p. 15): (1) as an instrument to achieve a (given) aim (instrumental), (2) to confer epistemic authority and thereby legitimacy (legitimating function), and (3) to substantiate already formed policy preferences (symbolic function). To illustrate, Christensen (2018, p. 293)points out that at least since the end of World War II, particular interest of policymakers accrues to economics, which has begun to bestow more legitimacy in policy advice than other forms of knowledge. This has been variously attributed to economics' (supposed) role in ensuring prosperity, and in economics bestowing an aura of rationality on decision-making (ibid.).

Even while there is a vast literature studying impact, the relative importance of different factors has not been established (Haynes et al., 2011). With few exceptions, the literature on the topic fails to clearly define or analyse the fundamental concepts ("research uptake", "impact", "policy advice") and the problems that are at stake. Notable counterexamples include works by Weiss (1979), Mitton et al. (2007) - who build upon Weiss's model -, and Blewden et al. (2010), whereas Cairney, along with various collaborators, has published extensively on the second issue (Cairney, 2016; Cairney, Oliver and Wellstead, 2016; Wellstead, Cairney and Oliver, 2018; Oliver and Cairney, 2019). The respective literature can be grouped as follows:

1) Problem diagnosis: Empirical evidence for the evidence-policy gap (mostly qualitative, i.e. interviews and surveys)

2) Problem solving: Recommendations for researchers on how to "bridge the evidence-policy gap"

3) Critique: The diagnosis of an evidence-policy-gap results from a normative problem definition and an analysis based on a rudimentary (common sense) understanding of policy processes

Here, we focus on the first and second group to distil the common denominator of the empirical work on why research uptake does (not) work. As Oliver and Cairney (2019) point out, the advice offered to academics wishing to engage with policy makers is frequently inconsistent. We would like to add to this the observation that the analyses of the science-policy interface are, if not inconsistent, then frequently uninformative. In fact, while there is now a large body of work documenting barriers to the use of scientific evidence in policy processes, taken at face value, most of the advice for overcoming barriers to uptake appears commonsensical and generic. Most of it uncritically assumes a gap between academia and policy, otherwise known as the evidence-policy gap (Ellen, Léon, et al., 2014), that "needs bridging" (Rajić, Young and McEwen, 2013), often going so far as "using the exact phrasing" (Oliver and Cairney, 2019, p. 3) to suggest (rather than demonstrate) that their advice will help foster research uptake. This literature further assumes that policy is rarely based on data, and that greater use of evidence will produce better outcomes, an assumption that remains empirically untested (Oliver and Cairney, 2019). At best, studies recommend process-related improvement (e.g., by reference to increasing transparency) (Ellen, Léon, et al., 2014). Empirical work is frequently case-based without contextualization of the multi-faceted processes underlying policy development (Oliver, Lorenc and Innvær, 2014, p. 4).

\section{Key factors in research uptake: Relationships, resources, and research skills}

Debates about the nature of the problems have spawned various literatures engaging with policy advice from empirical and theoretical standpoints. In drafting this section, we predominantly relied on four 
narrative reviews of barriers and facilitators for the use of evidence by policymakers (Cairney, Oliver, and Wellstead 2016; Liverani, Hawkins, and Parkhurst 2013; Oliver et al. 2014; Oliver, Lorenc, and Innvær 2014) that we amended by including empirical studies. Contacts and relationships (social capital) are reported throughout the literature as major facilitators of evidence use (Oliver et al., 2014, p. 7). According to Oliver et al. (2014, p. 4), timing and opportunity are the most important factors, along with (dis)trust and mutual (dis)respect. Policymakers seek information that is timely, relevant, credible, and available (Head, 2015, p. 7). Organisational factors such as (lack of) access to scientific results, (lack of) material and personnel resources and managerial support, and inflexible and non-transparent policy processes are mentioned frequently (ibid. 4 f.) Quality, relevance, and reliability of research as well as presentation formats act as facilitators (ibid. 6). However, accessible communication of research involves trade-offs: Clear writing makes research more digestible but at increased cost for researchers (Merlo et al., 2015).Respondents value researchers who exhibit competence (pragmatism)/reputation), integrity (faithful representation of research) and independence (more important to politicians), and benevolence/commitment (Oliver et al., 2017, p. 122). For research to be effective in policy making, a fundamental requirement is effective communication (e.g. Dodson, Geary and Brownson, 2015), a responsibility ascribed to researchers (e.g. Haynes et al., 2012). Lack of understanding/awareness of research on the part of policymakers was reported as a barrier (ibid. 6), as were (lack of) personal experience, values, and judgements. Respondents scarcely attribute lack of uptake to the policy process itself (Cairney, Oliver and Wellstead, 2016). Indeed, the literature often bemoans a general lack of reflection on policy processes (ibid.). Research uptake is further enabled/hampered by organizational constraints, influence of fads and trends on the policy process (Weiss, 1979), corruption and ideology as well as cultural beliefs (Haines, Kuruvilla and Borchert, 2004). The most frequent organizational barriers to research uptake were limited resources (financial or personnel), time constraints (to make decisions or participate in training), high staff turnover and institutional resistance towards change (Ellen, Lavis, et al., 2014). On the other hand, decision makers' willingness to create a culture of knowledge translation and to invest resources was mentioned as a facilitator.

In what follows, we identify key factors that the literature holds (not) to be conducive to research uptake: a) quality of relationships and informants, b) resources and access to research, c) communication formats and policy makers' research skills, and d) the policy context, and discrepancies in values, and goals. As we will demonstrate, the value proposition of Open Science directly or indirectly relates to several of these factors which suggests that the genericity of the analysis of barriers carries over to the proposition that Open Science will enhance research uptake.

a) Relationship quality and quality of informants: Relationship quality is a well-recognized research area. Collaboration between researchers and policymakers, along with relationships and skills, are the most frequently reported facilitators of research uptake (Oliver et al. (2014). (Long-term) Collaboration starting in the early stages of knowledge production is favoured by researchers and policymakers alike (Choi et al., 2016). Mutual mistrust is a well-researched barrier (Elliott and Popay, 2000; Choi et al., 2005; Gold, 2009; Haynes et al., 2012; Gollust, Seymour, Pany and Goss, 2017). Researchers are advised to build better communication channels and relationships with policymakers (Gold, 2009; Grimshaw et al., 2012; Head, 2015; Glied, Wittenberg and Israeli, 2018). While policy makers worry about bias in research, researchers qualify policy processes as biased (Gollust, Seymour, Pany, Goss, et $a l ., 2017)$. Positivism is thereby an artefact of requests for unbiased truth. The strategies employed by researchers to influence policy are likewise value-laden and cannot be understood solely as evidencebased (Cairney and Oliver 2017). Because researchers and policymakers belong to different communities (Löblová, 2018), the role of the knowledge broker has gained importance (Gagnon 2019) in facilitating knowledge transfer (Gluckman, Bardsley and Kaiser, 2021). Sustained dialogue between researchers and policymakers is essential for the development of researchers' perspectives, in-depth knowledge of the policy process, and credibility (Glied, Wittenberg and Israeli, 2018). This aspect of the problem mirrors the constraints posed by differences in timescales (Choi et al., 2005; Blewden, Carroll and Witten, 2010; Ellen et al., 2011; Ellen, Lavis and Shemer, 2016; Cairney and Rummery, 2018). The prevalence of informal contacts entails that science-policy interactions lack transparency. (Hermann et al., 2015). Policy-makers treat scientific input as an internal concern with the effect that recommendations by committees remain invisible. (Oliver et al., 2014) document an increasing amount of research stressing the serendipitous nature of the policy process which gives primacy to informal 
contacts. In these environments, formalized advice through contract research does not promote transparency, but shifting to research programs has boosted transparency regarding beneficiary institutions, funding amounts, topics and publication of results (Hermann et al., 2015).

Policymakers' advisors reside either inside or outside public bodies (Christensen, 2018, p. 295). The current knowledge transfer landscape includes a set of (more or less) formalized roles (Olesk, Kaal and Toom, 2019, p. 3). Policymakers trust government sources as well as advocacy, industry and lobby groups, and experts (Dodson, Geary and Brownson, 2015, p. 844). Policymakers trust their networks and personal contacts most for information (Oliver et al., 2017); academics are rarely represented in them (Oliver et al., 2017, p. 122). As their research awareness is low, policymakers prefer opinion leaders as information sources. Few academics participate directly in the decision-making process (ibid.). Policymakers prefer local experts, governmental agencies, and websites to academic publications. Policymakers predominantly seek (quantitative) data and statistics (Dodson, Geary and Brownson, 2015, p. 842), but also use other information which they consider relevant and timely (Oliver, Lorenc and Innvær, 2014).

b) Organizational factors and access to academic resources: Lack of resources is a frequent barrier to academic policy advice (Cairney, Oliver, and Wellstead 2016, 400). Resources are invested to the extent knowledge exchange is deemed profitable (Contandriopoulos et al., 2010, p. 462). Researchers tend to expect knowledge transfer to produce immediate results (Blewden, Carroll and Witten, 2010). However, the temporal structure of policy making is ill-attuned to academic influence (Kothari et al., 2011, p. 205) as timescales of policymaking are shorter than those of academia (Head, 2015). Time constraints keep policy makers from directly engaging with research. Timely access to good quality research is conducive to uptake, poor access and lack of timely research output are frequent barriers (Oliver et al., 2014), as is the short-term nature of research funding (Elliott and Popay, 2000, p. 467). Knowledge transfer is deeply embedded in organizational, institutional and policy contexts (Contandriopoulos et al., 2010, p. 468) which influence how relationships between academia and government evolve (Head, 2015, p. 7), but is not featured in tenure/promotion criteria (Gollust, Seymour, Pany, Goss, et al., 2017). Patterns of evidence use and management vary across domains and across organizational types (Head, 2016).

Access to information is important in research uptake (Oliver, Lorenc and Innvær, 2014). Policymakers need relevant research to make well-informed decisions (Cambon et al., 2017). Costs associated with access inhibit research uptake; public servants use their university affiliations to circumvent this (Olesk, Kaal and Toom, 2019). Research needs to be both accessible (to potential users) and acceptable (in terms of the evidence provided) (Merlo et al., 2015, p. 303). Accessibility enables timely use of evidence; acceptability can either mean scientific acceptability (valid methods, unbiased results, modelling assumptions), institutional acceptability (evidence meets the institutional needs of the decision maker), or ethical acceptability (Merlo et al., 2015). There are trade-offs between the accessibility and the acceptability of research findings such that the use of statistical apparatus might improve the acceptability of a certain evidence base it but only at the cost of decreasing its accessibility to nonexperts. External funding may similarly increase accessibility but harm scientific acceptability. (Merlo et al., 2015) The propensity of organisations for research uptake depends on formal and informational structures for organisational learning (Abekah-Nkrumah et al., 2018). Translation via up-to-date research syntheses that are easier to consume and less likely to be biased could help (Grimshaw et al., 2012). Systematic reviews are regarded as fundamental in transferring evidence from medical and health research to health policy making (Tugwell et al., 2006; Abekah-Nkrumah et al., 2018; Marquez et al., 2018), but even systematic reviews require translation (Haines, Kuruvilla and Borchert, 2004), making for the importance of intermediaries' (Gold, 2009). Formal structures within research-performing institutions along with mechanisms to make syntheses available could facilitate research uptake (Gold, 2009; Abekah-Nkrumah et al., 2018). Given policy-makers' preferences for personal contacts, the availability and accessibility of scholarly publications is of secondary concern (Oliver et al., 2017).

c) Communication formats and research skills: Scholarly communication via peer-reviewed publications is ill-attuned to the needs of policymakers who prefer personal contacts (Haynes et al., 2012). Potential experts are identified based on engagement with literature, through conferences, personal networks and reputation (e.g. past committee memberships), media presence, and sometimes through self-identification (Dodson, Geary and Brownson, 2015) (Haynes et al., 2012). Oral forms of 
communication are more commonly used than written material; the ability to communicate clearly and concisely is highly sought after. Policymakers prefer personal contacts; formal procedures to identify experts are rare (Oliver et al., 2017).

Policymakers involve such heterogeneous actors as politicians, public servants, administrators, lobbyists, and interest groups (Saretzki, 2019). Evidence helps decision makers to reduce uncertainty, but policymakers rely on beliefs and emotions in choosing a problem interpretation (Cairney, Oliver, and Wellstead 2016). Policymakers' abilities in finding and making sense of evidence facilitate research uptake (Oliver et al., 2014; Cambon et al., 2017). Policymakers struggle with knowledge management and have difficulties appraising research (Ellen et al., 2011; Head, 2015, p. 7), in addition to a lack of financial resources, knowledge, attitudes, and skills (Ellen et al., 2011). Because uptake depends on data interpretation and analysis skills, mere access to data and other research outputs (systematic reviews, individual studies, grey literature) is not sufficient (Lillefjell and Knudtsen, 2013).

d) Policy context and discrepancies in norms and goals: The policy context is fundamental for the use of evidence (Blewden, Carroll and Witten, 2010; Contandriopoulos et al., 2010; Glied, Wittenberg and Israeli, 2018; Krick, 2019). Policy making is an unpredictable, long-term, multilevel process involving networks of policymakers, paradigms, and norms in a quick succession of priorities (Cairney, Oliver, and Wellstead 2016, 400; Paul Cairney and Rummery 2018, 544). The inclusion of academics and interest groups into the policy process is subject to cultural differences (Hermann et al., 2015). Research needs to be policy-relevant in the first place to be considered by policymakers (Rose et al., 2019), but this is only a necessary (not a sufficient) condition. Policy-making and academia have different goals and success criteria (Holm and Ploug, 2015, p. 8). Policy is not driven by neutral scientific evidence. Policymakers are motivated by factors other than research evidence (Head, 2016, p. 474). The policy process is inherently political, involving interests and power relations and necessarily depending on policymakers' preferences, goals, and values (Kothari et al., 2011, p. 204; Head, 2016, p. 473). These deliberative aspects are difficult to account for in problem-centred analyses of knowledge transfer (Elliott and Popay, 2000, p. 467). Evidence pertains to ends and means (Head, 2016, p. 473), and needs to be embedded in action proposals (Contandriopoulos et al., 2010, p. 459). Researchers work with small, clearly defined problems; policymakers address problems holistically (Kothari et al., 2011, p. 204), discrepancies that make collaboration prone to conflict (e.g. Choi et al., 2005). Collaboration is not neutral; it works best when research goals match policy aims (Boyko, Kothari and Wathen, 2016). Discrepancies in norms and values influence how the potential for research uptake is perceived (Martin, Currie and Lockett, 2011);internal validity of information does not by itself influence the use of information (Contandriopoulos et al., 2010, p. 457). Research uptake therefore depends upon relevance to a given policy context (Weiss, 1979), legitimacy (of knowledge producers), and accessibility (Contandriopoulos et al., 2010, p. 460). Even where the impact of scientific evidence on policy advice is evident (e.g. Christensen, 2018), it is not clear whether changes in the culture of policy advice have an impact on policies. The same can be said for research more generally. In addition to having to answer questions of implementation, policymakers need to worry about being re-elected and striking compromise between competing groups. All these factors limit the extent to which policies can be evidence-based.

\section{Summary: What constitutes the Evidence-Policy-Gap?}

To sum up the previous discussion, the evidence-policy-gap is described in various ways:

1) failure to implement cost-effective health interventions and a corresponding need to promote interactions between researchers and policymakers (Haines, Kuruvilla and Borchert, 2004)

2) ways of enhancing the uptake of evidence-based interventions and the potential of scientific discoveries (Brownson and Jones, 2009; Choi et al., 2016)

3) (in)ability of public health services to integrate research findings into their decisions and operations (Dodson, Geary and Brownson, 2015; Cambon et al., 2017)

4) barriers and facilitators to evidence-informed decision-making (Elliott and Popay, 2000; Ellen, Léon, et al., 2014; Merlo et al., 2015) 
As Cairney and others have pointed out, the diagnosis implicitly assumes the existence of (only) two distinct groups (researchers and policymakers) with competing goals, values, and languages who should communicate but, for reasons developed in detail in the literature, don't succeed in doing so (e.g. Cairney, Oliver, and Wellstead 2016). The diagnosis is a methodical artefact in this perspective (Oliver, Lorenc and Innvær, 2014). As Cairney (2016) remarks, a focus on barriers leads to overlooking the normal workings of policy processes (Oliver, Lorenc and Innvær, 2014, p. 4). Research frequently describes single elements of the process (dissemination, sources of evidence, knowledge transfer) rather than the entire process (p. 5).

The upshot of this discussion is that the evidence-policy-gap diagnosis draws on an overly idealized, simplistic image of the policy process and that methodically assessing the impact of Open Science outputs on policy making is complicated by the fact that commissioned research is rarely published (Hermann et al., 2015). Additionally, there are no accessible (quantitative) data on research utilization. As has been shown, both Open Science-friendly literature as well as discussions of the evidence-policygap have described science predominantly as a linguistic phenomenon, resulting in a familiar problem diagnosis and similar appeals that access and uptake can be achieved by improving scholarly communication. 


\section{Discussion: Open Science at the science-policy interface - models of knowledge, models of policy}

Cairney and collaborators argue that the literature on the evidence-policy gap lacks the conceptual resources to describe how policy processes work. In a similar vein, (Gollust, Seymour, Pany, Goss, et al., 2017) point out that scientists and policy makers live in different worlds and often do not understand or appreciate the specifics (needs, requirements, logics) of the other (Haynes et al., 2012; Holm and Ploug, 2015; Gollust, Seymour, Pany, Goss, et al., 2017; Choi et al., 2005). ${ }^{2}$ Most of the literature on the evidence-policy gap implicitly assumes a "two cultures model", highlighting the

"differences in academic and political "cultures": language and jargon, longer scientific timescales, low incentives to engage, differing perceptions of scientific knowledge, and the relative need for scientists to challenge evidence (to ensure it is robust) but for policy makers to generate an image of policy certainty and reconcile evidence with well-established beliefs. There is also a perception that policy makers rely on personal experience, ad hoc links with experts, people they know and trust, and simple decision-making techniques and stories rather than the state-of-the-art in scientific research and sophisticated modeling systems (Lomas and Brown 2009, 906)". (Cairney, Oliver, and Wellstead 2016, 400)

This model turns on an - ultimately untenable - separation of two aspects of policy advice: Reducing uncertainty (Cairney, Oliver and Wellstead, 2016; Cairney and Oliver, 2017) versus reducing ambiguity and increasing clarity (Cairney and Oliver 2017). Cairney et al. (2016) further point out that a large proportion of the empirical work has focussed on a third aspect that concerns improving the flow of information between decision makers and researchers. The evidence-policy gap has thus been defined in terms of improving the evidence base, better communication of information, improved interaction with policymakers, better timing, and the use of knowledge brokers (Rose et al. 2019). If Cairney et al.'s (2016) analysis is correct, a deeper analysis of the way policy processes work is necessary to understand which aspects are amenable to influence. However, instead of attempting to understand policy processes, "researchers have directed their attention at how to increase their own outputs, rather than on understanding the processes behind policy change" (Oliver, Lorenc, and Innvær 2014, 3).

Policy models largely remain implicit; studies frequently rely on a linear understanding of the sciencepolicy interface. Likewise, the mechanisms by which scientific advice may benefit policy are rarely explicated. We suggest the following strategy to shed light on these assumptions: 1) We contrast two clusters of policy models 2) we discuss the compatibility of both approaches to policy making with propositions found in the reviewed literature to trace implicit assumptions about policy making and about the science-policy interface to reveal the outlines of a largely opaque understanding of policy processes implicit in the reviewed literature and to point out some inherent difficulties of the discussed approaches. Analytically, we contrast representative democracy with participatory democracy. According to the representative democracy model, policy making takes place primarily in (national, regional, municipal) parliaments and effectively boils down to decisions made by elected representatives in parliaments and respective assemblies. By contrast, participatory democracy involves policy arenas beyond legislative assemblies, even when the locus of deliberation is much broader in participatory democracy, involving actors who are not formally elected politicians.

Overall, the arguments presented in the reviewed literature resonate with the representative democracy model rather than with other models of policy making. This implicit choice of policy model has fundamental implications for understanding the evidence-policy gap. For research to be effective in policy making, researchers need to maintain their credibility and reputation (e.g. Haynes et al., 2012);

\footnotetext{
${ }^{2}$ Choi et al. 2005 argue in a similar vein that researchers and policymakers differ substantially in their goals, values, attitudes towards information, languages, perceptions of time, and career paths (e.g. Choi et al., 2005). If correct, this entails that researchers need to balance conflicting roles and identities when engaging with policymakers in policy contexts. These have been described with reference to Mertonian disinterestedness (Merton, 1973) as an attitude associated with argumentative rigour and rationality which bears the associated risk of policy irrelevance (Haynes et al., 2011).
} 
Oliver et al. 2017). Impartiality is a key characteristic for advice offered to legislating assemblies (Hilgartner 2000). Accordingly, policy advice needs to be framed as impartial to be acceptable.

Whereas the representation of partial interests is constitutive for policy making (Kothari et al., 2011, p. 204; Head, 2016, p. 473) especially for "competitive" representative democracies, this is not a viable option for scientists who ground their reputation in impartiality and feel committed to the ethos of impartial knowledge transfer (cf. Merton, see also Elliott and Popay, 2000, p. 467).

The situation results in considerable difficulties and ambiguities for researchers engaging in policy advice in terms of maintaining their role as impartial knowledge brokers, compromising their possibilities to influence political agenda setting or to openly promote specific policy goals. Efforts to this effect are therefore often framed in terms of knowledge deficits on the part of policy makers. Framing the evidence-policy gap as a knowledge problem allows researchers to promote normative policy goals while at the same time keeping these goals in the scientific domain and maintaining their authority over it. Policy makers on the other hand capitalise on the objectivity of scientific knowledge in order to present their normative positions as objective (Lundin et al. (2014), using scientific evidence to make arguments about ends and means (Head, 2016, p. 473). This intertwinement of science and policy leads us to propose a third analytical point.

We would like to suggest that the opacity of the policy making process in the reviewed literature exactly corresponds to the epistemological opacity of the scientific knowledge in question. By and large, the literature presents scientific knowledge as sacrosanct; scientific knowledge offered in the form of policy advice is taken for granted. Once published, the epistemic status (validity, credibility) of scientific knowledge is no longer questioned and can thus be delivered to policy makers, notwithstanding the fact that knowledge users might require translation. A deeper analysis of the tenets of the literature on research uptake therefore shows that positivist conceptions of knowledge correspond with a specific view of policy making as parliamentarian deliberation which in turn corresponds to a linear model of research uptake. There is an implicit assumption that solving policy problems requires, and science provides, impartial knowledge. This epistemological outlook remains largely implicit. By paying attention to what is conspicuously absent in the literature on the evidence-policy gap, this implicit position can be revealed. For instance, the literature never speaks to constructivist epistemologies (knowledge as co-constructed by researchers, policy makers and civil society actors). Equally, since it assumes a linear process involving two groups, the epistemic role of users, patient groups, (health) professionals or communities of practice is rarely touched upon. Likewise, influential interpretations of Open Science as the provision of open access to scientific outputs are indicative in omitting interpretations that foreground the accessibility of knowledge creation (public participation and engagement).

Similar to the affinities of the representative policy model with ideas of impartial knowledge and positivist epistemologies, there is an affinity of participatory models with conceptions of transdisciplinary knowledge . Corresponding epistemologies understand scientific knowledge as coconstructed, context-dependent and situated. It follows that for knowledge to be policy relevant, it is its creation (deliberation and implementation) and implementation need to be participatory processes. As we have attempted to show, the absence of contributions that ostensibly draw on participatory models and related forms of knowledge production is indicative of the implicit assumptions in the reviewed literature on the evidence-policy gap. 


\section{Conclusion}

Scientific policy advice is essential for policy makers, both in terms of addressing immediate issues and in terms of long-term planning. In public health in particular, critical accounts point to low rates of research uptake in the policy domain, a problem known as the evidence-policy-gap. In this paper, we examined whether Open Science could serve to bridge the evidence-policy gap and found claims to the effect that Open Science can boost the utilization of publicly available scientific results and data by policymakers (e.g. (Olesk, Kaal, and Toom 2019; Tennant, Jacques, and Collister 2016) to be unsubstantiated. There is little empirical evidence as to the effectiveness of Open Science practices with respect to the uptake of scientific results by policymakers.

These rather sobering findings raise further questions with respect to modelling the science-policy interface. As we have argued, expectations to the effect that Open Science will increase research uptake are often linked to a linear understanding of knowledge transfer. Thus, the hope of Open Science as a key enabler for more evidence-informed policy making was found to be driven by two assumptions: The first assumption concerns the role of analysis and rationality in policy making. While it is true that policy is not entirely value-driven, the inclusion of facts rests on deliberating the information content and how it fits in with policy goals. The second assumption concerns the modes of communication between what is considered to be two spheres. There, a large body of empirical literature has suggested that improving the uptake of scientific findings by policymakers hinges upon improving communication channels. As Open Science primarily seeks to reform academic communication, Open Science advocacy has argued in a similar vein that research uptake mainly rests upon the availability of evidence. We have seen how this picture underestimates the complexities of research uptake in the policy world, where a myriad of other factors may be at play, such as receptiveness to evidence (and the same is true for society more generally). The hopes of Open Science advocates turn on a linear understanding of policy advice where impartial experts supply elected (elite) representatives with impartial information via platform technology under conditions of consensual or undisputed policy goals and the availability of certified knowledge. Therefore, what is needed first and foremost is open access to these platforms. The twocultures model likewise assumes that effective communication can serve to bridge the gap.

We saw that influential interpretations regard Open Science primarily as an intervention into scientific communication, mandating research outputs be made available as early as possible within the research lifecycle. However, this narrow interpretation of Open Science falls short of the way current analyses model the science-policy interface. Interventions on one side of the interface are not enough to enhance uptake.

The two-cultures-model draws on a similar problem diagnosis and suggests ways to overcome barriers to knowledge uptake by legislators. Better communication is seen as key to fixing the problem. What is needed to enhance uptake are knowledge brokers capable of translating academic jargon to legislators and thereby broker between academia and the world of policy making (Pielke 2009). Despite the acknowledgement of divergent logics characterising the scientific and political domains it is striking that normativity is hardly seen to constitute incommensurability between science and policy making. If, however, language, temporality and cognitive barriers are viewed as the main problem, effective communication promises increased uptake of scientific knowledge in the policy domain.

Against this backdrop, we argue that the expectations in the benefits of Open Science in the policy domain are bound to the assumption of a linear transfer of knowledge. In light of recent approaches to the science-policy interface, the linear model appears rather outdated (Sokolovska et al. 2019). However, these approaches have also been criticized for failing to address the mechanisms of the policy process itself (Cairney 2016). Claims that the evidence-policy-gap can be closed by improving communication and other forms of interaction depends upon the linear model even while acknowledging its inherent limitations.

Recent literature on the science-policy interface observes considerable advancements beyond the linear model. In particular, deliberation with a broader set of stakeholders has been suggested as a more inclusive and effective form of knowledge integration in the policy domain. The discussions presented here hold few references to deliberative approaches as preferable forms of knowledge integration. 
Although the importance of personal communication with policymakers were frequently acknowledged, responsibility was largely identified with researchers' personal skills.

Reference to more recent theoretical developments of the science-policy interface where a broader inclusion of civil society actors is promoted were rare., as were references to knowledge integration as part of participatory approaches towards policy making (as outlined by e.g. Sokolovska et al. 2019). Despite its apparent relevance, the problematisation of the evidence-policy gap contains scant consideration of this quite substantial body of literature on the subject.

Against this backdrop, we conclude that claims of low research uptake in the policy domain (the evidence-policy gap) result from an implicit adherence to outdated models of the science-policy interface. It is therefore not surprising that Open Science practices were likewise found to have little impact upon uptake of scientific knowledge in the policy domain.

Nevertheless, we want to close by pointing out that Open Science could potentially contribute to policy making in other ways. To that end, however, it would be necessary to make implicit policy models as parliamentarian legislation as well as notions of positivist epistemologies Open Science adheres to explicit. If the policy model is expanded to include practices of deliberative and participatory policy, Open Science may have more to offer than what is now conceived almost exclusively as the provision of access to scientific outputs and more effective scholarly communication. This potential is even greater where participation is made possible from the outset of knowledge making processes. Scientific knowledge that is co-produced by users, practitioners and other stakeholders holds the potential of being policy relevant beyond legislative assemblies. It remains an open question how researchers who engage in various forms of policy advice should interpret their role in light of the normative dimensions of policy making. To wit, where policy goals cannot be taken for granted, conceptions of impartial knowledge are difficult to maintain. This discussion is linked to the discussion around the correct analysis of truth familiar from epistemology (whether correspondence, coherence, pragmatist, or consensus theories). All views have their merits and are valid in different situations. It would seem, then, that conceptions of knowledge and conceptions of the policy process are resources of the social actors involved. I.e., when researchers want to stress their credibility and impartiality, they need to resort to a positivistic model of science etc.

In conclusion, our analysis of the literature suggests the dominance of a particular framing of the sciencepolicy interface which corresponds to a particular epistemology. This approach largely interprets Open Science as the provision of access to scientific outputs and their effective communication while alternative views are conspicuously absent. We conclude that effectiveness of Open Science practices with respect to research uptake is for the most part a proposition that is not yet grounded in evidence. This is a shortcoming that extends to the effectiveness of enhanced communication and knowledge brokerage. Participatory approaches and the contribution of more inclusive forms of knowledge making have seen some success (e.g. Epstein 1996). However, they were not framed in terms of Open Science, but rather as deliberative democracy, participatory technology assessment, public engagement, or responsible research and innovation, respectively. 


\section{References}

Abekah-Nkrumah, G. et al. (2018) 'A review of the process of knowledge transfer and use of evidence in reproductive and child health in Ghana', Health Research Policy and Systems, 16. doi:10.1186/s12961-018-0350-9.

Albornoz, D. et al. (2017) Co-Constructing an Open and Collaborative Manifesto to Reclaim the Open Science Narrative, Expanding Perspectives on Open Science: Communities, Cultures and Diversity in Concepts and Practices. Edited by L. Chan and F. Loizides, pp. 293-304. doi:10.3233/978-1-61499769-6-293.

Albornoz, D. et al. (2020) 'Framing power: Tracing key discourses in open science policies', in 22nd International Conference on Electronic Publishing - Connecting the Knowledge Commons: From Projects to Sustainable Infrastructure, ELPUB 2018. Available at: https://www.scopus.com/inward/record.uri?eid=2-s2.0-

85085471179\&partnerID=40\&md5=4d9a26ae9080a4c38e819ac062070c9c.

Amorim, R.C. et al. (2017) 'A comparison of research data management platforms: architecture, flexible metadata and interoperability', Universal Access in the Information Society, 16(4), pp. 851-862. doi:10.1007/s10209-016-0475-y.

Bardi, A., Casarosa, V. and Manghi, P. (2018) 'The European Project OpenUP: OPENing UP New Methods, Indicators and Tools for Peer Review, Impact Measurement and Dissemination of Research Results', in Serra, G. and Tasso, C. (eds) Digital Libraries and Multimedia Archives, Ircdl 2018. Berlin: Springer-Verlag Berlin, pp. 240-248. doi:10.1007/978-3-319-73165-0_24.

Beck, J. et al. (2018) 'Publishing peer review materials [version 1; referees: 2 approved]', F1000Research, 7. Available at: https://doaj.org (Accessed: 7 February 2019).

Blewden, M., Carroll, P. and Witten, D.K. (2010) 'The use of social science research to inform policy development: case studies from recent immigration policy', Kōtuitui: New Zealand Journal of Social Sciences Online, 5(1), pp. 13-25. doi:10.1080/1175083X.2010.498087.

Boyko, J.A., Kothari, A. and Wathen, C.N. (2016) 'Moving knowledge about family violence into public health policy and practice: a mixed method study of a deliberative dialogue', Health research policy and systems, 14, p. 31.

Brownson, R.C. and Jones, E. (2009) 'Bridging the gap: translating research into policy and practice', Preventive Medicine, 49(4), pp. 313-315. doi:10.1016/j.ypmed.2009.06.008.

Bugaje, M. and Chowdhury, G. (2018) 'Identifying Design Requirements of a User-Centered Research Data Management System', in Lecture Notes in Computer Science, Information Systems and Applications, incl. Internet/Web, and HCI, pp. 335-347. doi:10.1007/978-3-030-04257-8_35. 
Cairney, P. (2016) The politics of evidence-based policy making. Palgrave Macmillan.

Cairney, P. and Oliver, K. (2017) 'Evidence-based policymaking is not like evidence-based medicine, so how far should you go to bridge the divide between evidence and policy?', Health Research Policy and Systems, 15(1), pp. 1-11. doi:10.1186/s12961-017-0192-х.

Cairney, P., Oliver, K. and Wellstead, A. (2016) 'To Bridge the Divide between Evidence and Policy: Reduce Ambiguity as Much as Uncertainty', Public Administration Review, 76(3), pp. 399-402. doi:10.1111/puar.12555.

Cairney, P. and Rummery, K. (2018) 'Feminising Politics to Close the Evidence-Policy Gap: The Case of Social Policy in Scotland', Australian Journal of Public Administration, 77(4), p. 542. doi:10.1111/1467-8500.12266.

Cambon, L. et al. (2017) 'Evaluation of a knowledge transfer scheme to improve policy making and practices in health promotion and disease prevention setting in French regions: a realist study protocol', Implementation Science, 12, p. 83. doi:10.1186/s13012-017-0612-x.

Carroll, M.W. (2015) ‘Sharing Research Data and Intellectual Property Law: A Primer’, PLOS Biology, 13(8), p. e1002235. doi:10.1371/journal.pbio.1002235.

Carrozza, T.J. and Brieva, S.S. (2015) 'Who owns the Atoms? Nanotechnology and Intellectual Property Rights', Íconos, 19(52), pp. 163-180.

Caso, R. and Ducato, R. (2014) Intellectual Property, Open Science and Research Biobanks. SSRN Scholarly Paper ID 2511602. Rochester, NY: Social Science Research Network. Available at: https://papers.ssrn.com/abstract=2511602 (Accessed: 12 November 2019).

Choi, B.C.K. et al. (2005) 'Can scientists and policy makers work together?', Journal of Epidemiology and Community Health, 59(8), p. 632. doi:10.1136/jech.2004.031765.

Choi, B.C.K. et al. (2016) 'Bridging the gap between science and policy: an international survey of scientists and policy makers in China and Canada', Implementation Science, 11. doi:10.1186/s13012016-0377-7.

Christensen, J. (2018) 'Economic knowledge and the scientization of policy advice', Policy Sciences, 51(3), pp. 291-311. doi:10.1007/s11077-018-9316-6.

Contandriopoulos, D. et al. (2010) 'Knowledge Exchange Processes in Organizations and Policy Arenas: A Narrative Systematic Review of the Literature', The Milbank Quarterly, 88(4), pp. 444-483. doi:10.1111/j.1468-0009.2010.00608.x.

Cox, A.M. et al. (2017) 'Developments in research data management in academic libraries: Towards an understanding of research data service maturity', Journal of the Association for Information Science and Technology, 68(9), pp. 2182-2200. doi:10.1002/asi.23781. 
Dodson, E.A., Geary, N.A. and Brownson, R.C. (2015) 'State legislators' sources and use of information: bridging the gap between research and policy', Health Education Research, 30(6), pp. 840848. doi:10.1093/her/cyv044.

Ellen, M.E. et al. (2011) 'Determining research knowledge infrastructure for healthcare systems: A qualitative study', Implementation Science, 6(1). doi:10.1186/1748-5908-6-60.

Ellen, M.E., Léon, G., et al. (2014) 'Barriers, facilitators and views about next steps to implementing supports for evidence-informed decision-making in health systems: a qualitative study', Implementation science : IS, 9, p. 179. doi:10.1186/s13012-014-0179-8.

Ellen, M.E., Lavis, J.N., et al. (2014) 'Health systems and policy research evidence in health policy making in Israel: what are researchers' practices in transferring knowledge to policy makers?', Health research policy and systems / BioMed Central, 12, p. 67. doi:10.1186/1478-4505-12-67.

Ellen, M.E. et al. (2018) 'How is the use of research evidence in health policy perceived? A comparison between the reporting of researchers and policy-makers', Health Research Policy and Systems, 16(1). doi:10.1186/s12961-018-0345-6.

Ellen, M.E., Lavis, J.N. and Shemer, J. (2016) 'Examining the use of health systems and policy research in the health policymaking process in Israel: views of researchers', Health Research Policy and Systems, 14. doi:10.1186/s12961-016-0139-7.

Elliott, H. and Popay, J. (2000) 'How are policy makers using evidence? Models of research utilisation and local NHS policy making', Journal of Epidemiology and Community Health, 54(6), p. 461. doi:10.1136/jech.54.6.461.

Etzkowitz, H. and Leydesdorff, L. (1990) 'The Second Academic Revolution: The Role of the Research University in Economic Development', in Cozzens, S.E. et al. (eds) The Research System in Transition. Dordrecht and Boston \& London: Kluwer, pp. 109-124.

Fecher, B. and Friesike, S. (2014) 'Open Science: One Term, Five Schools of Thought', in Bartling, S. and Friesike, S. (eds) Opening Science. Cham: Springer International Publishing, pp. 17-47. doi:10.1007/978-3-319-00026-8_2.

Fell, M.J. (2019) 'The Economic Impacts of Open Science: A Rapid Evidence Assessment', Publications, 7(3), p. 46. doi:10.3390/publications7030046.

Felt, U. (2003) O.P.U.S. - Optimizing Public Understanding of Science and Technology. Final Report. Vienna: Department of Philosophy of Science and Social Studies of Science, University of Vienna.

Gibbons, M. et al. (1994) The new production of knowledge. The dynamics of science and research in contemporary societies. London et al.: Sage Publications. 
Giffels, J. (2010) 'Sharing Data is a Shared Responsibility', Science and Engineering Ethics, 16(4), pp. 801-803. doi:10.1007/s11948-010-9230-6.

Gilmore, R.O. et al. (2017) 'Progress toward openness, transparency, and reproducibility in cognitive neuroscience', Annals of the New York Academy of Sciences, 1396(1), pp. 5-18. doi:10.1111/nyas.13325.

Glied, S., Wittenberg, R. and Israeli, A. (2018) 'Research in government and academia: the case of health policy', Israel Journal of Health Policy Research, 7(1), pp. 1-8. doi:10.1186/s13584-018-02303.

Gluckman, P.D., Bardsley, A. and Kaiser, M. (2021) 'Brokerage at the science-policy interface: from conceptual framework to practical guidance', Humanities and Social Sciences Communications, 8(1), pp. 1-10. doi:10.1057/s41599-021-00756-3.

Gold, M. (2009) 'Pathways to the Use of Health Services Research in Policy', Health Services Research, 44(4), pp. 1111-1136. doi:10.1111/j.1475-6773.2009.00958.x.

Gollust, S.E., Seymour, J.W., Pany, M.J., Goss, A., et al. (2017) 'Mutual distrust: Perspectives from researchers and policy makers on the research to policy gap in 2013 and recommendations for the future', INQUIRY: The Journal of Health Care Organization, Provision, and Financing, 54, pp. 1-11. doi:10.1177/0046958017705465.

Gollust, S.E., Seymour, J.W., Pany, M.J. and Goss, A. (2017) 'Mutual distrust: Perspectives from researchers and policy makers on the research to policy gap in 2013 and recommendations for the future', Inquiry (United States), 54. doi:10.1177/0046958017705465.

Graham, I.D. et al. (2006) 'Lost in knowledge translation: Time for a map?', JOURNAL OF CONTINUING EDUCATION IN THE HEALTH PROFESSIONS, 26(1), pp. 13-24. doi:10.1002/chp.47.

Grahe, J.E. et al. (2020) 'Open Science Promotes Diverse, Just, and Sustainable Research and Educational Outcomes', Psychology Learning and Teaching-Plat, 19(1), pp. 5-20. doi:10.1177/1475725719869164.

Grimshaw, J.M. et al. (2012) 'Knowledge translation of research findings', Implementation science: IS, 7, p. 50. doi:10.1186/1748-5908-7-50.

Haines, A., Kuruvilla, S. and Borchert, M. (2004) 'Bridging the implementation gap between knowledge and action for health', Bulletin of the World Health Organization, 82(10), pp. 724-731; discussion 732. Harding, S. (2002) 'Must the Advance of Science Advance Global Inequality?', International Studies Review, 4(2), pp. 87-105. doi:10.1111/1521-9488.00256. 
Haynes, A.S. et al. (2011) "From "our world" to the "real world": Exploring the views and behaviour of policy-influential Australian public health researchers', Social Science \& Medicine (1982), 72(7), pp. 1047-1055. doi:10.1016/j.socscimed.2011.02.004.

Haynes, A.S. et al. (2012) 'Identifying Trustworthy Experts: How Do Policymakers Find and Assess Public Health Researchers Worth Consulting or Collaborating With?', PLoS ONE, 7(3), p. e32665. doi:https://doi.org/10.1371/journal.pone.0032665.

Head, B.W. (2015) 'Relationships between Policy Academics and Public Servants: Learning at a Distance?', Australian Journal of Public Administration, 74(1), pp. 5-12. doi:10.1111/14678500.12133 .

Head, B.W. (2016) 'Toward More “Evidence-Informed” Policy Making?', Public Administration Review, 76(3), pp. 472-484. doi:10.1111/puar.12475.

Hermann, A.T. et al. (2015) 'Cultural Imprints on Scientific Policy Advice: Climate science-policy interactions within Austrian neo-corporatism', Environmental Policy and Governance, 25(5), p. 343. doi:10.1002/eet.1674.

Hillyer, R. et al. (2017) 'Framing a Situated and Inclusive Open Science: Emerging Lessons from the Open and Collaborative Science in Development Network', in Expanding Perspectives on Open Science: Communities, Cultures and Diversity in Concepts and Practices.

Holm, S. and Ploug, T. (2015) 'The use of empirical evidence in formulating reproductive policy advice and policy', Monash Bioethics Review, 33(1), pp. 7-17. doi:10.1007/s40592-015-0020-4.

Ibanez, L., Avila, R. and Aylward, S. (2006) 'Open Source and Open Science: how it is changing the medical imaging community', in 3rd IEEE International Symposium on Biomedical Imaging: Nano to Macro, 2006. 3rd IEEE International Symposium on Biomedical Imaging: Nano to Macro, 2006., pp. 690-693. doi:10.1109/ISBI.2006.1625010.

Jasanoff, S. (2005) Designs on Nature. Science and Democracy in Europe and the United States. Princeton and Oxford: Princeton University Press.

Kitchin, R. (2014) 'Big Data, new epistemologies and paradigm shifts', Big Data \& Society, 1(1), p. 2053951714528481. doi:10.1177/2053951714528481.

Kothari, A. et al. (2011) 'Indicators at the interface: Managing policymaker-researcher collaboration', Knowledge Management Research and Practice, 9(3), pp. 203-214. doi:10.1057/kmrp.2011.16.

Krick, E. (2019) 'Creating participatory expert bodies. How the targeted selection of policy advisers can bridge the epistemic-democratic divide', European Politics and Society, 20(1), pp. 101-116. doi:10.1080/23745118.2018.1515865. 
Lavis, J.N. et al. (2003) 'How Can Research Organizations More Effectively Transfer Research Knowledge to Decision Makers?', The Milbank Quarterly, 81(2), pp. 221-248. doi:10.1111/14680009.t01-1-00052.

Leonelli, S. (2016) Data-centric Biology. A philosophical study. Chicago, London: The University of Chicago Press.

Leonelli, S., Spichtinger, D. and Prainsack, B. (2015) 'Sticks and carrots: encouraging open science at its source', Geo, 2(1), pp. 12-16. doi:10.1002/geo2.2.

Lillefjell, M. and Knudtsen, M.S. (2013) 'From knowledge to action in public health management: Experiences from a Norwegian context', Scandinavian Journal of Public Health, 41(8), pp. 771-777. doi:10.1177/1403494813496600.

Liverani, M., Hawkins, B. and Parkhurst, J.O. (2013) 'Political and institutional influences on the use of evidence in public health policy. A systematic review', PloS One, 8(10), p. e77404. doi:10.1371/journal.pone.0077404.

Löblová, O. (2018) 'Epistemic communities and experts in health policy-making', European Journal of Public Health, 28(suppl_3), pp. 7-10. doi:10.1093/eurpub/cky156.

Lundin, M. and Öberg, P. (2014) 'Expert knowledge use and deliberation in local policy making', Policy Sciences, 47(1), pp. 25-49. doi:10.1007/s11077-013-9182-1.

Maasen, S. and Weingart, P. (eds) (2005) Democratization of Expertise?: Exploring Novel Forms of Scientific Advice in Political Decision-Making. Springer Netherlands (Sociology of the Sciences Yearbook). doi:10.1007/1-4020-3754-6.

Marquez, C. et al. (2018) 'Enhancing the uptake of systematic reviews of effects: what is the best format for health care managers and policy-makers? A mixed-methods study', Implementation Science, 13, p. 84. doi:10.1186/s13012-018-0779-9.

Martin, G., Currie, G. and Lockett, A. (2011) 'Prospects for knowledge exchange in health policy and management: institutional and epistemic boundaries', Journal of Health Services Research \& Policy, 16(4), pp. 211-217. doi:10.1258/jhsrp.2011.010132.

McKiernan, E.C. et al. (2016) 'How open science helps researchers succeed', eLife, 5. doi:10.7554/eLife.16800.

Merlo, G. et al. (2015) 'Bridging the gap: exploring the barriers to using economic evidence in healthcare decision making and strategies for improving uptake', Applied Health Economics and Health Policy, 13(3), pp. 303-309. doi:10.1007/s40258-014-0132-7.

Mirowski, P. (2018) 'The future(s) of open science', Social Studies of Science, 48(2), pp. 171-203. doi:https://doi.org/10.1177/0306312718772086. 
Mitton, C. et al. (2007) 'Knowledge Transfer and Exchange: Review and Synthesis of the Literature', The Milbank Quarterly, 85(4), pp. 729-768. doi:10.1111/j.1468-0009.2007.00506.x.

Münch, R. (2014) Academic Capitalism. London, New York: Routledge.

Nagasaka, K., Böcher, M. and Krott, M. (2016) 'Are forest researchers only scientists? Case studies on the roles of researchers in Japanese and Swedish forest policy processes', Forest Policy and Economics, 70, pp. 147-154. doi:10.1016/j.forpol.2016.06.006.

Nielsen, M. (2013) Reinventing Discovery: The New Era of Networked Science. Princeton: Princeton University Press.

Nosek, B.A. et al. (2015) 'Promoting an open research culture', Science, 348(6242), pp. 1422-1425. doi:10.1126/science.aab2374.

Olesk, A., Kaal, E. and Toom, K. (2019) 'The possibilities of Open Science for knowledge transfer in the science-policy interface', Journal of Science Communication, 18(3). doi:10.22323/2.18030203.

Oliver, K. et al. (2014) 'A systematic review of barriers to and facilitators of the use of evidence by policymakers', BMC Health Services Research, 14(2), pp. 1-12. doi:10.1186/1472-6963-14-2.

Oliver, K. et al. (2017) 'Identifying public health policymakers' sources of information: comparing survey and network analyses', European Journal of Public Health, 27, pp. 118-123. doi:10.1093/eurpub/ckv083.

Oliver, K. and Cairney, P. (2019) 'The dos and don'ts of influencing policy: a systematic review of advice to academics', Palgrave Communications, 5(1), pp. 1-11. doi:10.1057/s41599-019-0232-y.

Oliver, K., Lorenc, T. and Innvær, S. (2014) 'New directions in evidence-based policy research: a critical analysis of the literature', Health Research Policy and Systems, 12(34), pp. 1-12. doi:10.1186/14784505-12-34.

Owen, R. and Pansera, M. (2019) 'Responsible innovation and responsible research and innovation', in Handbook on science and public policy. Northampton, MA: Edward Elgar Publishing, pp. 26-48.

Panisset, U. et al. (2012) 'Implementation research evidence uptake and use for policy-making', Health Research Policy and Systems, 10, p. 20. doi:10.1186/1478-4505-10-20.

Pielke, R.A. (2007) The Honest Broker. Making Sense of Science in Policy and Politics. Cambridge, New York, Melbourne, Madrid, Cape Town, Singapore, Sao Paulo: Cambridge University Press.

Pisani, E. et al. (2010) 'Time for fair trade in research data', Lancet (London, England), 375(9716), pp. 703-5. doi:10.1016/S0140-6736(09)61486-0.

Piwowar, H.A. (2011) 'Who Shares? Who Doesn't? Factors Associated with Openly Archiving Raw Research Data', PLOS ONE, 6(7), p. e18657. doi:10.1371/journal.pone.0018657. 
Piwowar, H.A., Day, R.S. and Fridsma, D.B. (2007) 'Sharing Detailed Research Data Is Associated with Increased Citation Rate', PLOS ONE, 2(3), p. e308. doi:10.1371/journal.pone.0000308.

Piwowar, H.A. and Vision, T.J. (2013a) 'Data reuse and the open data citation advantage', PeerJ, 1, p. e175. doi:10.7717/peerj.175.

Piwowar, H.A. and Vision, T.J. (2013b) 'Data reuse and the open data citation advantage', PeerJ, 1, p. e175. doi:10.7717/peerj.175.

Pöschl, U. (2012) 'Multi-Stage Open Peer Review: Scientific Evaluation Integrating the Strengths of Traditional Peer Review with the Virtues of Transparency and Self-Regulation', Frontiers in Computational Neuroscience, 6. doi:10.3389/fncom.2012.00033.

Rajić, A., Young, I. and McEwen, S.A. (2013) 'Improving the utilization of research knowledge in agrifood public health: A mixed-method review of knowledge translation and transfer', Foodborne Pathogens and Disease, 10(5), pp. 397-412. doi:10.1089/fpd.2012.1349.

Ram, K. (2013) 'Git can facilitate greater reproducibility and increased transparency in science', Source Code for Biology and Medicine, 8(1), p. 7. doi:10.1186/1751-0473-8-7.

Reichmann, S., Wieser, B. and Ross-Hellauer, T. (2020) 'ON-MERRIT D5.1 Scoping Report: Open Science Outputs in Policy-Making and Public Participation'. doi:10.5281/zenodo.3875055.

Richterich, A. (2018) The Big Data Agenda: Data Ethics and Critical Data Studies. University of Westminster Press.

Rose, D.C. et al. (2019) 'Improving the use of evidence in legislatures: the case of the UK Parliament', Evidence \& Policy [Preprint]. Available at: http://centaur.reading.ac.uk/88497/ (Accessed: 16 April 2020).

Ross-Hellauer, T. (2017) 'What is open peer review? A systematic review', F1000Research, 6, p. 588. doi:10.12688/f1000research.11369.2.

Saretzki, T. (2019) 'Evidence-based policy-making? The meaning of scientific knowledge in policy processes', Zeitschrift für Evidenz, Fortbildung und Qualität Im Gesundheitswesen, 144-145, pp. 7883. doi:10.1016/j.zefq.2019.05.008.

Schaeffer, V. (2019) 'The use of material transfer agreements in academia: A threat to open science or a cooperation tool?', Research Policy, 48(9), p. UNSP 103824. doi:10.1016/j.respol.2019.103824.

von Schomberg, R. (2019) 'Why Responsible Innovation', in The International Handbook on Responsible Innovation. A Global resource. Cheltenham: Edward Elgar Publishing.

Shanahan, D.R. and Olsen, B.R. (2014) 'Opening peer-review: the democracy of science', Journal of Negative Results in BioMedicine, 13(1), p. 2. doi:10.1186/1477-5751-13-2. 
Slaughter, S. and Rhoades (2010) Academic Capitalism and the New Economy. Markets, State, and Higher Education. Baltimore, MD: The Johns Hopkins University Press.

Sokolovska, N., Fecher, B. and Wagner, G.G. (2019) 'Communication on the Science-Policy Interface: An Overview of Conceptual Models', Publications, 7(4), p. 64. doi:10.3390/publications7040064.

Stilgoe, J. (2013) 'Why Responsible Innovation?', in Responsible Innovation. Making the responsible emergence of science and innovation in society. Chichester: Wiley, pp. xi-xv.

Suber, P. (2012) Open Access. MIT Press. Cambridge, MA.

Tennant, J., Jacques, D. and Collister, L. (2016) 'The academic, economic and societal impacts of Open Access: an evidence-based review', F1000Research. Edited by L. Collister, 5. doi:10.12688/f1000research.8460.1.

Toelch, U. and Ostwald, D. (2018) 'Digital open science-Teaching digital tools for reproducible and transparent research', PLOS Biology, 16(7), p. e2006022. doi:10.1371/journal.pbio.2006022.

Tugwell, P. et al. (2006) 'Systematic reviews and knowledge translation', Bulletin of the World Health Organization, 84(8), pp. 643-651. doi:10.2471/blt.05.026658.

Vale, R.D. and Hyman, A.A. (2016) 'Priority of discovery in the life sciences', eLife, 5, p. e1693. doi:10.7554/eLife.16931.

Vicente-Saez, R. and Martinez-Fuentes, C. (2018) 'Open Science now: A systematic literature review for an integrated definition', Journal of Business Research, 88, pp. 428-436. doi:10.1016/j.jbusres.2017.12.043.

Weingart, P. (1983) 'Verwissenschaftlichung der Gesellschaft - Politisierung der Wissenschaft', Zeitschrift für Soziologie, 12(3), pp. 225-241. doi:10.1515/zfsoz-1983-0303.

Weingart, P. (1999) 'Scientific expertise and political accountability: paradoxes of science in politics', Science and Public Policy, 26(3), pp. 151-161. doi:10.3152/147154399781782437.

Weiss, C.H. (1979) ‘The Many Meanings of Research Utilization', Public Administration Review, 39(5), pp. 426-431. doi:10.2307/3109916.

Wellstead, A., Cairney, P. and Oliver, K. (2018) 'Reducing ambiguity to close the science-policy gap', Policy Design and Practice, 1(2), pp. 115-125. doi:10.1080/25741292.2018.1458397.

Wynne, B. (1993) 'Public uptake of science: a case for institutional reflexivity', Public Understanding of Science, 2(4), pp. 321-337. doi:10.1088/0963-6625/2/4/003.

Wynne, B. (1995) 'Public Understanding of Science', in Handbook of Science and Technology Studies. Thousand Oaks, London, New Delhi: Sage Publications, pp. 361-388. 